\title{
The Impact of Supplemental Macular Carotenoids in Alzheimer's Disease: A Randomized Clinical Trial
}

\author{
John M. Nolan ${ }^{\mathrm{a}, *}$, Ekaterina Loskutova ${ }^{\mathrm{a}}$, Alan Howard ${ }^{\mathrm{b}, \mathrm{c}}$, Riona Mulcahy ${ }^{\mathrm{d}}$, Rachel Moran ${ }^{\mathrm{a}}$, Jim Stack ${ }^{\mathrm{a}}$, \\ Maggie Bolger ${ }^{\mathrm{d}}$, Robert F. Coen ${ }^{\mathrm{e}}$, Jessica Dennison ${ }^{\mathrm{a}}$, Kwadwo Owusu Akuffo ${ }^{\mathrm{a}}$, Niamh Owens ${ }^{\mathrm{a}}$, \\ Rebecca Power ${ }^{\mathrm{a}}$, David Thurnham ${ }^{\mathrm{f}}$ and Stephen Beatty ${ }^{\mathrm{a}}$ \\ ${ }^{a}$ Macular Pigment Research Group, Department of Chemical and Life Sciences, Waterford Institute of Technology, \\ Waterford, Ireland \\ ${ }^{\mathrm{b}}$ Howard Foundation, Cambridge, UK \\ ${ }^{\mathrm{c}}$ Downing College, University of Cambridge, Cambridge, UK \\ ${ }^{\mathrm{d}}$ University Hospital Waterford, Age-Related Care Unit, Waterford, Ireland \\ ${ }^{\mathrm{e}}$ Mercer's Institute for Successful Ageing, St. James's Hospital, Dublin, Ireland \\ ${ }^{\mathrm{f}}$ Northern Ireland, Centre for Food and Health (NICHE), University of Ulster, Coleraine, UK
}

Accepted 15 October 2014

\begin{abstract}
.
Background: Patients with Alzheimer's disease (AD) exhibit significantly less macular pigment (MP) and poorer vision when compared to control subjects.

Objective: To investigate supplementation with the macular carotenoids on MP, vision, and cognitive function in patients with $\mathrm{AD}$ versus controls.

Methods: A randomized, double-blind clinical trial with placebo and active arms. 31 AD patients and 31 age-similar control subjects were supplemented for six months with either Macushield (10 mg meso-zeaxanthin [MZ]; $10 \mathrm{mg}$ lutein [L]; $2 \mathrm{mg}$ zeaxanthin [Z]) or placebo (sunflower oil). MP was measured using dual-wavelength autofluorescence (Heidelberg Spectralis ${ }^{\circledR}$ ). Serum L, Z, and MZ were quantified by high performance liquid chromatography. Visual function was assessed by best corrected visual acuity and contrast sensitivity (CS). Cognitive function was assessed using a battery of cognition tests, including the Cambridge Neuropsychological Test Automated Battery (CANTAB).

Results: Subjects on the active supplement (for both AD and non-AD controls) exhibited statistically significant improvement in serum concentrations of $\mathrm{L}, \mathrm{Z}, \mathrm{MZ}$, and MP $(p<0.001$, for all $)$ and also CS at $(p=0.039)$. Also, for subjects on the active supplement, paired samples $t$-tests exhibited four significant results (from five spatial frequencies tested) in the AD group, and two for the non-AD group, and all indicating improvements in CS. We found no significant changes in any of the cognitive function outcome variables measured ( $p>0.05$, for all).

Conclusion: Supplementation with the macular carotenoids (MZ, Z, and L) benefits patients with AD, in terms of clinically meaningful improvements in visual function and in terms of MP augmentation.
\end{abstract}

Keywords: Age-related macular degeneration, Alzheimer's disease, cognitive function, contrast sensitivity, lutein, mesozeaxanthin, randomized clinical trial, visual function, zeaxanthin

\footnotetext{
${ }^{*}$ Correspondence to: Professor John Nolan, Macular Pigment Research Group, Vision Research Centre, Carriganore House, Waterford Institute of Technology, West Campus, Carriganore, Waterford, Ireland. Tel.: +353 51 834074; E-mail: jmnolan@wit.ie.
}

\section{INTRODUCTION}

We have recently reported in the Carotenoids and Age-Related Dementia Study (CARDS, report 1) that patients with mild to moderate Alzheimer's disease 
(AD) exhibit significantly less macular pigment (MP), poorer vision, and a higher occurrence of age-related macular degeneration (AMD; another age-related disorder), when compared to control subjects [1].

MP, which is made up of the dietary carotenoids lutein $(\mathrm{L})$, zeaxanthin $(\mathrm{Z})$, and meso-zeaxanthin $(\mathrm{MZ})$ [2], is found exclusively at the central macula and can be measured in vivo $[3,4]$. Of note, the macula (the central part of the retina) is part of the central nervous system, and it is this specialized part of the retina that is responsible for central and detailed vision [5]. We know that the macular carotenoids, via their shortwavelength (blue) light filtering [6] and antioxidant properties [7, 8], play a protective role in AMD [9]. We also know that MP is positively related to visual function [10], and that enrichment of MP with nutritional supplements containing the macular carotenoids improves visual function in normal subjects (i.e., subjects without retinal disease) [11] and in subjects with early stages of AMD [12-15]. Indeed, the optical properties of MP, which include its preferential absorption of short-wavelength (blue) light, is likely to explain the visual benefits noted in previous clinical trials [16].

Of interest, we know from previous work that $\mathrm{L}$ and $\mathrm{Z}$ are present in the brain, including in the cerebellum, pons, and frontal and occipital cortices [17-19], and that their concentrations in the brain are positively correlated with retinal concentrations of these nutrients in primates [18] including humans [19]. Furthermore, there is a growing body of evidence suggesting a positive relationship between MP levels and cognitive function in humans [20-22] and Johnson et al. have reported that supplementation with the macular carotenoids impacts positively on cognitive function in older women [23].

Given the growing body of evidence showing that oxidative stress and inflammation contribute to cognitive impairment $[24,25]$ and AD pathogenesis [26, 27], it is plausible that carotenoids in the brain could protect against such stresses, given their proven antioxidant [7, $8]$ and anti-inflammatory properties [28, 29]. It has also been suggested that the carotenoids may play a beneficial role by enhancing gap junctional communication in the brain [30-32].

In summary, we have already reported that patients with $\mathrm{AD}$ have significantly less MP, lower serum concentrations of $\mathrm{L}$ and $\mathrm{Z}$, poorer vision, and a higher occurrence of AMD when compared to control subjects. Also, there is a biologically plausible rationale, supported by a growing body of scientific evidence, which suggests that enrichment of retinal and brain nutrition with the carotenoids $\mathrm{L}, \mathrm{Z}$, and $\mathrm{MZ}$ will protect and enhance cognitive function in humans. This study was conducted to investigate the impact of supplementation with the macular carotenoids on MP (primary outcome measure), and vision and cognitive function (secondary outcome measures) in patients with $\mathrm{AD}$ compared with controls of similar age, and is the first study to do so.

\section{MATERIALS AND METHODS}

\section{Study design and subject recruitment}

This clinical trial began in January 2013 (i.e., the first subject visit) and ended in September 2013 (i.e., last subject six month visit).

31 patients with mild to moderate AD (predominantly moderate) were recruited (through the Age-Related Care Unit at University Hospital Waterford [UHW]) into the study. Subjects recruited into this group (the AD group) were eligible if they had mild to moderate $\mathrm{AD}$, which was defined as having an average Mini-Mental State Examination (MMSE) score of 14 to 24 with documented difficulties in other domains, such as carrying out activities of daily living, or behavioral changes. Subjects were excluded if they were currently taking supplements containing the macular carotenoids, or if they had done so over the previous 12 months. Other screening tests to check for eligibility included the clock drawing test and semantic fluency score. Co-morbid diagnoses were documented, including vascular risk factors and diabetes. Current medications were verified including cholinesterase inhibitors and glutamate receptor antagonists. Social histories were documented and collateral histories were collated from a family member or carer for all patients. Non-contrast computed tomography (CT) brain scan was performed to rule out radiological evidence of stroke disease.

Of note, $10(32 \%)$ had also participated in the cross-sectional study previously reported by our group (CARDS 1) [1] and 21 (68\%) were newly recruited. Importantly, the subjects that had already participated in CARDS 1 were re-examined at baseline of this study because of the time difference between the crosssectional examination and the start of this clinical trial. Subjects with AD were randomly allocated, in a double-blind fashion to a supplement consisting of either Macushield ${ }^{\mathrm{TM}}$ (Macuvision Europe Ltd. Blythe Valley Innovation Centre, Central Boulevard, Blythe Valley Business Park, Solihull B90 8AJ, United Kingdom) ( $n=16$, active supplement containing $10 \mathrm{mg} \mathrm{MZ;}$ $10 \mathrm{mg} \mathrm{L} ; 2 \mathrm{mg} \mathrm{Z})$ or placebo ( $n=15$, sunflower oil). 
The intervention and placebo supplements were identical in external appearance and therefore the two treatments were indistinguishable from each other.

An equal number $(n=31)$ of age-similar controls free of $\mathrm{AD}$ (the none-AD Group) were similarly allocated to Macushield $(n=15)$ or Placebo $(n=16)$. Subjects were eligible for this group if they were aged (years) $\geq 65$. Subjects were excluded if they were currently taking supplements containing the macular carotenoids, or if they had done so over the previous 12 months.

Main study visits were at baseline and after six months of supplementation. All measurements were performed at the Vision Research Centre, Carriganore House, Waterford, Ireland. This clinical trial facility offers a very efficient and calm environment to conduct clinical trials. For consistency, all measurements were performed by the same researcher (EL) who was suitably trained on all aspects and technologies for this clinical trial.

Significant efforts were made to ensure subject compliance to the study supplements. Compliance was assessed on an ongoing basis (house visits and phone calls to care givers) by the study nurse (MB) for the AD subjects, and by the researchers (NO and EL) for the control subjects (mainly phone calls directly to the subjects). In addition, compliance was assessed by examining pill sleeves at the six month visit and by assessing serum carotenoid response using high performance liquid chromatography (HPLC, see below). The code was broken at 6 months, and the statistical analysis was performed by the Study Statistician (JS) and Principal Investigator (JMN). The results of this analysis are presented below. The methodology used to measure MP, visual function and cognitive function has already been described in detail (see CARDS report 1), so only a brief account of each method is presented below.

\section{Ethics}

The project was conducted in accordance with full sensitivity to the ethical requirements of the subjects recruited. The study objectives and methodology complied fully with the widely recognized international text and codes of practice such as the Declaration of Helsinki. A protocol was developed specifically for this study by the Principal Investigator (JMN) and Consultant Geriatrician (RM) at UHW to ensure that informed consent was obtained appropriately, and in keeping with the ethical code germane to obtaining consent from vulnerable subjects (which includes patients with AD). Ethical approval was granted from the local
Waterford South East (of Ireland) Region Ethics Committee prior to the study commencing.

\section{Demographic, medical, ophthalmic, and lifestyle assessment}

A demographic, medical, ophthalmic, and lifestyle case history was obtained for each subject at baseline. Body mass index (BMI) was calculated $\left(\mathrm{kg} / \mathrm{m}^{2}\right)$ with subject height (m) measured with the Leicester Height Measure, and weight $(\mathrm{kg})$ measured with the SECA weighing scales (SECA, Birmingham, UK). Smoking status was classed as either current smoker (i.e., smoked $\geq 100$ cigarettes in lifetime and at least one cigarette within the last 12 months) or non-smoker (everybody else). Exercise was assessed by calculating the total exercise for any sporting activity measured as minutes per week. Diabetes was assessed by self-report and also by measuring HbA1c in blood (analysis conducted offsite at Biomnis Ireland, Three Rock Road, Sandyford Business Estate, Dublin 18, Ireland).

\section{Cognitive function assessment}

Cognition was assessed using a selection of validated measures. The MMSE was used to measure the severity of cognitive impairment. A semantic fluency score was obtained using "Animal" as the category (as many exemplars as possible in one minute) and phonemic fluency was measured using the 'FAS Test' (as many words as possible starting with each letter, one minute per letter) [33]. Also, three tasks were chosen from the Cambridge Neuropsychological Test Automated Battery (CANTAB) [34]. All were administered using a finger-operated touch-screen tablet PC using a set of scripted instructions. The Paired Associates Learning task was selected to assess visual learning and memory [35]. A modified version of the Verbal Recognition Memory task was selected to assess verbal learning and memory [33]. In the modified version a free recall format was used instead of a recognition format. The CANTAB Motor Screening Task was used to assess motor speed and accuracy by instructing the subject to touch the center of a series of crosses that are presented on the screen [36].

\section{Best corrected visual acuity and contrast sensitivity}

The eye with best corrected visual acuity (BCVA) was selected as the study eye for vision testing. If both eyes had the same BCVA, the right one was selected. 
BCVA was measured with a computerized LogMAR ETDRS test chart (Test Chart 2000 Xpert; Thomson Software Solutions) viewed at 4 meters (m). The Sloan Early Treatment Diabetic Retinopathy Study (ETDRS) letterset was used for this test. Letter contrast sensitivity (CS) was assessed using the computerized LogMAR ETDRS test chart (Test Chart 2000 Pro; Thomson Software Solutions) at five different spatial frequencies $(1.2,2.4,6.0,9.6,15.15 \mathrm{cpd})$ [37]. Both these methods have been described in more detail elsewhere [10, 38, 39].

\section{Retinal photograph assessment}

45 degree monoscopic color photographs, centered on the macula, were taken in both eyes using a Zeiss Visucam 200 (Carl Zeiss Meditec AG, Jena, Germany). Retinal photographs were assessed for the presence or absence of early AMD, in accordance with the International Classification and Grading System for Age-Related Macular Degeneration by a consultant ophthalmologist (SB) with a special interest in retinal disease and with a published track record in grading this condition [40, 41]. In brief, the presence of soft drusen and/or hypo-/hyper-pigmentary changes at the macula were classed as early AMD.

\section{Macular pigment measurement}

MP was measured using the Heidelberg Spectralis ${ }^{\circledR}$ HRA+OCT Multicolor (Heidelberg Engineering GmbH, Heidelberg, Germany). This new technology utilizes confocal scanning laser ophthalmoscopy (cSLO) imaging with diode lasers and uses dualwavelength autofluorescence (AF) for measuring MP $[4,42]$. Dual-wavelength AF in this device uses two excitation wavelengths, one that is well-absorbed by MP (488 nm, blue), and one that is not well absorbed by the pigment $(518 \mathrm{~nm}$, green). Of note, the AF method utilized in this study has previously been compared with the customized heterochromatic flicker photometry (cHFP) technique for measuring MP, and the measurements recorded from these two devices exhibited excellent concordance [4]. However, the physical (objective) AF device was deemed more appropriate for this study, because patients with AD might not have been able to use the subjective (non-physical) cHFP device.

The Heidelberg Spectralis ${ }^{\circledR}$ AF method provides an image of MP across its spatial profile, but here we report just central MP (at 0.23 degrees eccentricity) and MP volume (calculated as MP average times the area under the curve out to 8 degrees eccentricity).

\section{Dietary intake of carotenoids}

A subject's weekly intake of carotenoid-rich foods (eggs, broccoli, corn, dark leafy vegetables) was inputted into the "L/Z screener" to give a carotenoidbased diet score. The $\mathrm{L}$ and $\mathrm{Z}$ values used in the screener were those reported by Perry et al. [43]. This method of assessing and controlling for dietary intake of carotenoids has been used with success elsewhere [12]. Values are weighted for frequency of intake of the food and for bioavailability of $\mathrm{L}$ and $\mathrm{Z}$ within these foods. A ranking score reflecting the relative intakes (representing arbitrary units) was generated and used in analysis. For the $\mathrm{AD}$ subject, dietary habits were confirmed by a family member or carer.

\section{Serum carotenoid assessment}

Non-fasting blood samples were collected in $9 \mathrm{ml}$ vacuette tubes containing a ' $Z$ Serum Sep Clot Activator'. The blood samples were allowed to clot at room temperature for approximately $30 \mathrm{~min}$ and then centrifuged at $2700 \mathrm{rpm}$ for $10 \mathrm{~min}$ in a Gruppe GC 12 centrifuge (Desaga Sarstedt) to separate the serum from the whole blood. The resulting serum samples were stored at circa $-80^{\circ} \mathrm{C}$ until the time of batch analysis using HPLC.

First, the serum samples were analysed for $\mathrm{L}$ and total $\mathrm{Z}$ (co-eluted $\mathrm{Z}$ and MZ) using a reversed-phase HPLC method (Assay 1 , for details of method see publication by Nolan et al. [1]). The mixed $\mathrm{Z}$ fraction was automatically collected from Assay 1 using an Agilent 1260 fraction collector. The eluent was dried under a solvent concentrator (MiVac, GeneVac, Mason Technologies, Dublin, Ireland) and analyzed on Assay 2 for quantification of $\mathrm{Z}$ and MZ (Assay 2, for details of method see publication by Thurnham et al. [44]).

\section{Statistics}

The statistical packages IBM SPSS version 21 was used for statistical analysis. Random numbers (for the allocation of subjects to active supplement or placebo) were generated in Minitab version 16; block randomization was used. This study was very close in design to a $2^{2}$ factorial design (two factors each at two levels: Macushield/Placebo and AD/Control) with 15 subjects per cell. Such a study has statistical power of $81 \%$ to detect a main effect of 0.75 standard deviations, and power of $70 \%$ to detect an interaction effect of the same magnitude, at the 5\% level of significance [45].

Outcome variables analyzed included serum carotenoids, MP, visual function measures, and 
cognitive function measures. Between-group differences in these outcome variables at baseline (e.g., AD versus controls) were analyzed using Independent Samples $t$-tests or chi-squared tests as appropriate. Differences at baseline in demographic and lifestyle variables were also investigated, and controlled for in subsequent analyses, as appropriate.

The main focus of the present study was the investigation of change in the outcome variables over time (i.e., from baseline to six months). In other words, did supplementing with Macushield lead to improvements in these outcome variables, relative to the Placebo, and did the supplement work differently for AD and control subjects? Both of these research questions were addressed using Repeated Measures Analysis of Variance, with supplement [Macushield versus Placebo] and Group [AD yes/no] as between-subjects factors, and age and diet score as covariates. These covariates were included in the analyses because age and diet score were significantly different between AD and controls at baseline. For some cognitive scores, the assumptions required for Repeated Measures Analysis of Variance were violated, and in these cases we resorted to informal comparisons of change in score between $\mathrm{AD}$ and control subjects and between supplements.

In reporting findings in tables and figures, however, we considered that it would be more informative to report the results of paired $t$-tests, separately within each Supplement/Group patient category.

The 5\% level of significance was used throughout all analyses, without adjustment for multiple comparisons. On standard assumptions (5\% level of significance, two-tailed tests), the paired $t$-test subgroup analyses reported here, with about 15 subjects in each subgroup, had adequate power (82\%) to detect "large" effect sizes ( 0.8 standard deviations, on Cohen's definition [46]). In general, however, it should be borne in mind that this small exploratory study was under-powered for the detection of smaller effect sizes and for the other analyses reported.

\section{RESULTS}

\section{Baseline}

Table 1 below presents baseline statistics for the $\mathrm{AD}$ and control groups. Of note, the sample and data presented here is slightly different to our already published cross-sectional paper (CARDS 1), given that the sample was not precisely the same for CARDS 1 and CARDS 2. However, the conclusions are the same. We confirm that, at baseline in CARDS 2, AD subjects have significantly lower MP, poorer vision, poorer cognitive function, and a significantly higher prevalence of AMD, when compared to the control group.

Although we had attempted, when recruiting subjects for this study, to match the AD and control groups in terms of age, it can be seen in Table 1 that the AD group is significantly older (on average), and we therefore controlled for age in any analysis comparing outcome variables in $\mathrm{AD}$ and control groups. We also adjusted for diet score, the other variable which differed significantly at baseline between AD and control groups.

\section{Dropouts}

\section{Control group}

All 16 subjects on placebo completed their six month study visit, whereas there were 2 dropouts (n61 and n68) in the active (Macushield) group, resulting in 13 subjects in this arm of the study. Reasons given for dropout include: logistical difficulties (e.g., transport) and did not want to continue (willingness to participate).

\section{$A D$ group}

12 subjects on placebo completed their six month study visit and there were 3 dropouts. Reasons for dropout include: logistical difficulties and did not want to continue (ADCD7 and ADN33); moved to nursing home and could not continue (ADN30). Also, 12 subjects in the active (Macushield) group completed their six month visit and there were 4 dropouts. Reasons for dropout include: logistical difficulties and did not want to continue (ADCD13, ADN22, ADN35, and ADN36).

\section{Compliance}

All subjects returned their capsule box and sleeves at their six month assessment visit. Assessment of capsule sleeves indicated that all subjects were consuming the supplements over the six-month study period. Also, serum carotenoid response confirmed that subjects in the active group were consuming the carotenoid intervention and that subjects in the placebo group exhibited no change in their serum carotenoid concentrations.

\section{Changes from baseline to six months}

Serum concentrations of lutein, zeaxanthin, and meso-zeaxanthin after six months of supplementation

In the Repeated Measures Analysis of change in serum L, the within-subjects Time*Supplement 
Table 1

Demographic, lifestyle, vision, and cognition data of the $\mathrm{AD}$ and control subjects at baseline

\begin{tabular}{|c|c|c|c|}
\hline Variables & $\mathrm{AD}(n=31)$ & Control $(n=31)$ & Sig. \\
\hline \multicolumn{4}{|l|}{ Demographic and Health } \\
\hline Age (years) & $80 \pm 7.8$ & $76 \pm 6.6$ & 0.031 \\
\hline Body mass index $\left(\mathrm{Kg} / \mathrm{m}^{2}\right)$ & $24.6 \pm 5.8$ & $26.4 \pm 3.4$ & 0.174 \\
\hline Exercise (total minutes of exercise per week) & $174 \pm 218$ & $226 \pm 16$ & 0.304 \\
\hline Diet (estimated lutein and zeaxanthin intake) & $16 \pm 8$ & $24 \pm 14$ & 0.008 \\
\hline Serum lutein $(\mu \mathrm{mol} / \mathrm{L})$ & $0.232 \pm 0.113$ & $0.297 \pm 0.179$ & 0.104 \\
\hline Serum zeaxanthin $(\mu \mathrm{mol} / \mathrm{L})$ & $0.051 \pm 0.035$ & $0.074 \pm 0.042$ & 0.03 \\
\hline Education (total years in education) & $11 \pm 4$ & $14 \pm 4$ & 0.003 \\
\hline Smoking (\% current) & $8.60 \%$ & $9.70 \%$ & 0.88 \\
\hline Gender (\% female) & $58 \%$ & $42 \%$ & 0.203 \\
\hline \multicolumn{4}{|l|}{$\begin{array}{l}\text { Vision } \\
\text { Vistinat }\end{array}$} \\
\hline MP 0.23 & $0.41 \pm 0.21$ & $0.57 \pm 0.17$ & 0.002 \\
\hline MP vol & $4074 \pm 2585$ & $6326 \pm 2258$ & 0.001 \\
\hline BCVA & $88.9 \pm 11.4$ & $95.8 \pm 8.4$ & 0.009 \\
\hline $\mathrm{CS} 1.2(\mathrm{cpd})$ & $1.49 \pm 0.23$ & $1.75 \pm .22$ & $<0.001$ \\
\hline $\mathrm{CS} 2.4$ (cpd) & $1.47 \pm 0.25$ & $1.79 \pm 0.21$ & $<0.001$ \\
\hline CS6.0 (cpd) & $1.19 \pm 0.31$ & $1.42 \pm 0.24$ & 0.004 \\
\hline CS9.6 (cpd) & $0.94 \pm 0.30$ & $1.18 \pm 0.26$ & 0.005 \\
\hline AMD (\% with AMD) & $48.00 \%$ & $16.00 \%$ & 0.007 \\
\hline \multicolumn{4}{|l|}{ Cognition } \\
\hline MMSE & $19 \pm 3.7$ & $29 \pm 1.7$ & $<0.001$ \\
\hline Semantic fluency score & $6.0 \pm 3.2$ & $15.4 \pm 5.2$ & $<0.001$ \\
\hline Phonemic fluency score & $15.7 \pm 10.3$ & $32.5 \pm 13.8$ & $<0.001$ \\
\hline VRM (phase 1) & $1.4 \pm 1.2$ & $5.1 \pm 2.6$ & $<0.001$ \\
\hline VRM (phase 2) & $2.5 \pm 1.6$ & $7.3 \pm 2.7$ & $<0.001$ \\
\hline VRM (phase 3) & $3.4 \pm 2.1$ & $8.1 \pm 2.9$ & $<0.001$ \\
\hline VRM Delayed Recall & $0.4 \pm 1.2$ & $6.6 \pm 3.3$ & $<0.001$ \\
\hline VRM Savings Score & $0.1 \pm 0.2$ & $0.8 \pm 0.5$ & $<0.001$ \\
\hline PAL (total errors adjusted) & $136 \pm 14.5$ & $69 \pm 39$ & $<0.001$ \\
\hline PAL (total errors adjusted 6 shapes) & $28.5 \pm 5.8$ & $17.7 \pm 10.7$ & $<0.001$ \\
\hline PAL Stages Complete & $0.5 \pm 0.9$ & $4 \pm 1.7$ & $<0.001$ \\
\hline PAL Patterns Reached & $2.5 \pm 0.9$ & $7.2 \pm 4.9$ & $<0.001$ \\
\hline PAL First Trial Memory Score & $0.8 \pm 2.3$ & $9.7 \pm 5.5$ & $<0.001$ \\
\hline
\end{tabular}

Data displayed are mean \pm standard deviation for interval data and percentages for categorical data. Variables, variables analyzed in the study; $\mathrm{AD}$, subjects recruited into the study confirmed as having mild to moderate Alzheimer's disease; Control, subjects free of mild to moderate $\mathrm{AD}$ and of similar age to the AD subjects; Sig., the statistical difference ( $p$ value) between $\mathrm{AD}$ and control subjects assessed using independent samples $t$-tests or chi-squared depending on the variable of interest; Exercise, total exercise for any sporting activity measured as minutes per week; Diet, estimate of dietary intake of $\mathrm{L}$ and $\mathrm{Z}$; Serum lutein, serum concentrations of lutein in $\mu \mathrm{mol} / \mathrm{L}$; Serum zeaxanthin, serum concentrations of zeaxanthin in $\mu \mathrm{mol} / \mathrm{L}$; Smoking, current (smoked $\geq 100$ cigarettes in lifetime and at least one cigarette within the last 12 months) or non-smoking (smoked $\leq 100$ cigarettes in lifetime and none within the last 12 months); MP 0.23 , central macular pigment measured at 0.23 degrees eccentricity measured using the Heidelberg Spectralis ${ }^{\circledR}$. MP vol, a volume of MP calculated as MP average times the area under the curve out to 8 degrees eccentricity (measured using the Heidelberg Spectralis ${ }^{\circledR}$ ); BCVA, best corrected visual acuity; CS 1.2, CS 2.4, CS 6.0, and CS 9.6 = letter contrast sensitivity measured using the Thomson Software Solutions at 1.2, 2.4, 6.0, and 9.6 cycles per degree; AMD; age-related macular degeneration; MMSE, Mini-Mental State Examination; Semantic fluency score, a semantic fluency (categorical verbal fluency) score obtained from the number of animals named by the subject in 1 minute; Phonemic fluency score, a phonemic fluency (word fluency) score generated by the total number of words produced for the each of the letters F, A, and S, in 1 minute. MOT (mean latency), motor screening task measures the subject's speed of response; MOT (mean error), motor screening task measures the accuracy of the subject's pointing at cross targets; VRM (phase 1), VRM (phase 2), VRM (phase 3), Verbal Free Recall Memory immediate, three consecutive trials; VRM Delayed Recall, Verbal Free Recall Memory of the previous words after a delay period; VRM Savings Score, Delayed verbal recall divided by phase 3 immediate recall; PAL, Paired Associates Learning test which measure visual memory and new learning of the subjects; PAL (total errors adjusted), the adjusted score and includes an adjustment made for any stages not reached, allowing it to be comparable to all subjects even if the task was ended prematurely due to cognitive limitation; PAL (total errors adjusted 6 shapes), total errors made at the 6-pattern stage, adjusted for subjects who did not reach this stage; PAL Stages Complete, The number of stages successfully completed; PAL Patterns Reached, The number of patterns on the last problem in the task that the subject completed successfully; PAL First Trial Memory Score, The number of patterns correctly located after the first trial, summed across the stages completed.

interaction effect was significant $(p<0.001)$. Neither the Time*Group interaction $(p=0.65)$ nor the Time*Supplement*Group interaction $(p=0.97)$ was significant. Thus, there was a significant increase in serum L concentrations after 6 months for subjects on the active (Macushield) supplement compared with 
subjects on the placebo supplement, no significant difference over time between $\mathrm{AD}$ and controls, and no evidence that the supplement worked differently over time for $\mathrm{AD}$ versus controls.

Similar results were obtained for serum concentrations of $\mathrm{Z}$, in that the Time*Supplement effect was significant $(p=0.007)$, but not the others; and for MZ ( $p<0.001$ for Time*Supplement interaction). Thus, in short, we report that the active supplement significantly increases serum concentrations of $\mathrm{L}, \mathrm{Z}$ and $\mathrm{MZ}$, and it does so for both $\mathrm{AD}$ and control subjects.

These findings are presented in Table 2 and Fig. 1. In Table 2, all subjects on the active supplement (Macushield) exhibit significantly increased serum concentrations of $\mathrm{L}, \mathrm{Z}$, and $\mathrm{MZ}$, in both control and AD subjects at six months.

The placebo categories exhibit no significant change over this time period, with the exception of a statistically significant increase in serum concentrations of $\mathrm{L}$ in the $\mathrm{AD}$ group. Although this increase observed in the placebo group, for subjects with $\mathrm{AD}$, was statistically significant, it was small (only 17\%) compared to the large increase $(291 \%)$ observed in the active (Macushield) group for subjects with AD.

\section{Macular pigment at baseline and after six months of supplementation}

In the Repeated Measures Analysis of change in MP (at $0.23^{\circ}$ eccentricity), the within-subjects Time*Supplement interaction effect was significant $(p<0.001)$. Neither the Time*Group interaction $(p=0.92)$ nor the Time*Supplement*Group interaction $(p=0.39)$ was significant. Thus, there was a significant increase in central MP after 6 months, for subjects on the active supplement compared with subjects on the placebo supplement, no significant difference over time between AD and controls, and no evidence that the supplement worked differently over time for $\mathrm{AD}$ and controls.

Table 2

Serum concentrations of lutein, zeaxanthin meso-zeaxanthin at baseline and following six months of supplementation with either active or placebo intervention

\begin{tabular}{|c|c|c|c|c|c|c|}
\hline Group & Intervention & Measurement & Mean \pm SD at baseline & Mean \pm SD at six months & $\%$ Change & Sig. \\
\hline Control & placebo & serum L $(\mu \mathrm{mol} / \mathrm{L})$ & $0.319 \pm 0.188$ & $0.280 \pm 0.118$ & -12 & 0.381 \\
\hline Control & active & serum $L(\mu \mathrm{mol} / \mathrm{L})$ & $0.288 \pm 0.177$ & $1.05 \pm 0.361$ & +265 & $\mathrm{p}<0.001$ \\
\hline $\mathrm{AD}$ & placebo & serum $\mathrm{L}(\mu \mathrm{mol} / \mathrm{L})$ & $0.174 \pm 0.057$ & $0.203 \pm 0.074$ & +17 & $\mathbf{0 . 0 3 5}$ \\
\hline $\mathrm{AD}$ & active & serum $\mathrm{L}(\mu \mathrm{mol} / \mathrm{L})$ & $0.261 \pm 0.142$ & $1.02 \pm 0.655$ & +291 & $\mathrm{p}<0.001$ \\
\hline Control & placebo & serum $\mathrm{Z}(\mu \mathrm{mol} / \mathrm{L})$ & $0.082 \pm 0.047$ & $0.07 \pm 0.030$ & -15 & 0.321 \\
\hline Control & active & serum $\mathrm{Z}(\mu \mathrm{mol} / \mathrm{L})$ & $0.068 \pm 0.036$ & $0.126 \pm 0.04$ & +85 & 0.003 \\
\hline $\mathrm{AD}$ & placebo & serum Z ( $\mu \mathrm{mol} / \mathrm{L})$ & $0.042 \pm 0.024$ & $0.062 \pm 0.035$ & +48 & 0.145 \\
\hline $\mathrm{AD}$ & active & serum $\mathrm{Z}(\mu \mathrm{mol} / \mathrm{L})$ & $0.048 \pm 0.035$ & $0.109 \pm 0.076$ & +127 & 0.02 \\
\hline Control & placebo & serum MZ $(\mu \mathrm{mol} / \mathrm{L})$ & 0 & 0 & - & - \\
\hline Control & active & serum MZ $(\mu \mathrm{mol} / \mathrm{L})$ & 0 & $0.082 \pm 0.059$ & - & 0.001 \\
\hline $\mathrm{AD}$ & placebo & serum MZ $(\mu \mathrm{mol} / \mathrm{L})$ & 0 & 0 & - & - \\
\hline $\mathrm{AD}$ & active & serum MZ $(\mu \mathrm{mol} / \mathrm{L})$ & 0 & $0.081 \pm 0.089$ & - & 0.009 \\
\hline
\end{tabular}

Data displayed are mean \pm standard deviation. \% change, the calculated percentage change from baseline to six months, calculated as baseline value minus the six month value divided by baseline value, multiplied by 100 ( $-=$ negative change and $+=$ positive change); Sig., the $p$ value for paired-sample $t$ testing between baseline and six months for each group split by intervention; AD, Alzheimer's disease; Active, Macushield ${ }^{\text {TM}}$ : $10 \mathrm{mg}$ lutein, $10 \mathrm{mg}$ meso-zeaxanthin, and $2 \mathrm{mg}$ zeaxanthin; Placebo, sunflower oil.
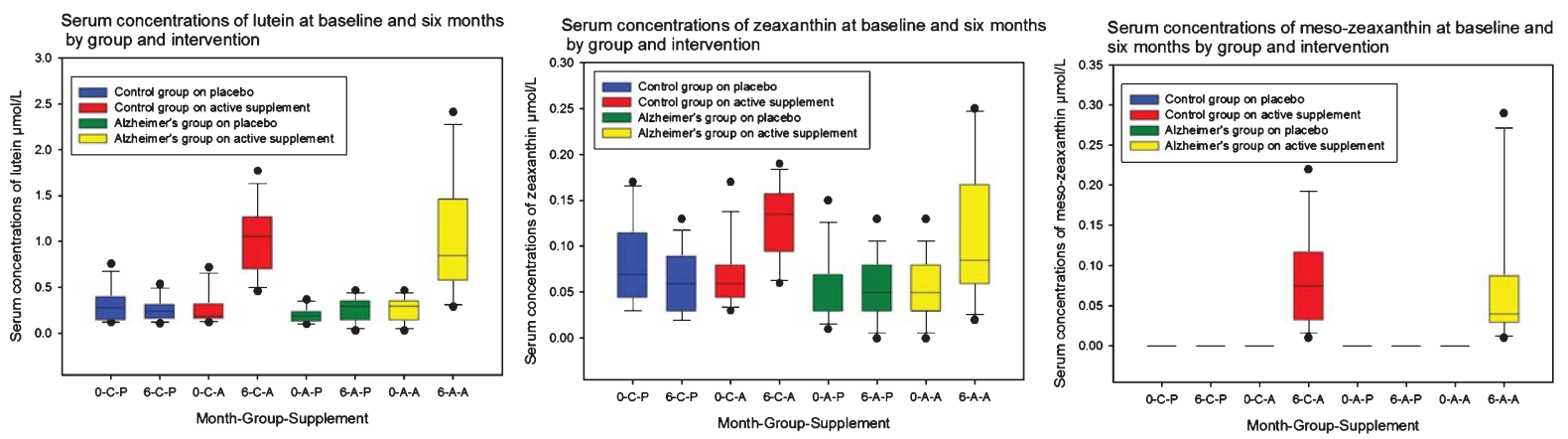

Fig. 1. Serum concentrations of lutein, zeaxanthin, and meso-zeaxanthin at baseline and six months by group and intervention. 0 , baseline; 6 , six months; C, control group; A, Alzheimer's group; P, placebo supplement; A, active supplement. 
Similar results were obtained for MP volume, in that only the Time*Supplement effect is significant $(p<0.001)$. Thus, in short, we report that the supplement works to increase MP, both centrally and across the spatial profile, and it does so for both $\mathrm{AD}$ and non-AD (control) subjects.

These findings are presented in Table 3 and Fig. 2. In Table 3, all subjects on the active supplement

Table 3

Macular pigment at baseline and following six months of supplementation with either active or placebo intervention

\begin{tabular}{|c|c|c|c|c|c|c|}
\hline Group & Intervention & Measurement & Mean \pm SD at baseline & Mean \pm SD at six months & $\%$ Change & Sig. \\
\hline Control & placebo & MP at $0.23^{\circ}$ & $0.58 \pm 0.18$ & $0.54 \pm 0.18$ & -7 & 0.300 \\
\hline Control & active & $\mathrm{MP}$ at $0.23^{\circ}$ & $0.58 \pm 0.18$ & $0.68 \pm 0.19$ & +17 & 0.002 \\
\hline $\mathrm{AD}$ & placebo & $\mathrm{MP}$ at $0.23^{\circ}$ & $0.40 \pm 0.17$ & $0.38 \pm 0.18$ & -5 & 0.86 \\
\hline $\mathrm{AD}$ & active & $\mathrm{MP}$ at $0.23^{\circ}$ & $0.41 \pm 0.26$ & $0.48 \pm 0.19$ & +17 & 0.009 \\
\hline Control & placebo & MP volume & $6543 \pm 2150$ & $6473 \pm 2131$ & -1 & 0.394 \\
\hline Control & active & MP volume & $6593 \pm 2116$ & $8291 \pm 2692$ & +26 & $\mathrm{p}<0.001$ \\
\hline $\mathrm{AD}$ & placebo & MP volume & $4008 \pm 2084$ & $4327 \pm 1948$ & +7 & 0.304 \\
\hline $\mathrm{AD}$ & active & MP volume & $3804 \pm 2255$ & $5408 \pm 3130$ & +42 & 0.001 \\
\hline
\end{tabular}

Data displayed are mean \pm standard deviation. \% change, the calculated percentage change from baseline to six months, calculated as baseline value minus the six month value divided by baseline value, multiplied by 100 ( $-=$ negative change and $+=$ positive change); Sig., the $p$ value for paired-sample $t$ testing between baseline and six months for each group split by intervention; MP at $0.23^{\circ}$, macular pigment at 0.23 degrees eccentricity; MP volume, MP average times the area under the curve out to 8 degrees eccentricity; AD, Alzheimer's disease; active, Macushield $^{\mathrm{TM}}: 10 \mathrm{mg}$ lutein, $10 \mathrm{mg}$ meso-zeaxanthin, and $2 \mathrm{mg}$ zeaxanthin; placebo, sunflower oil.
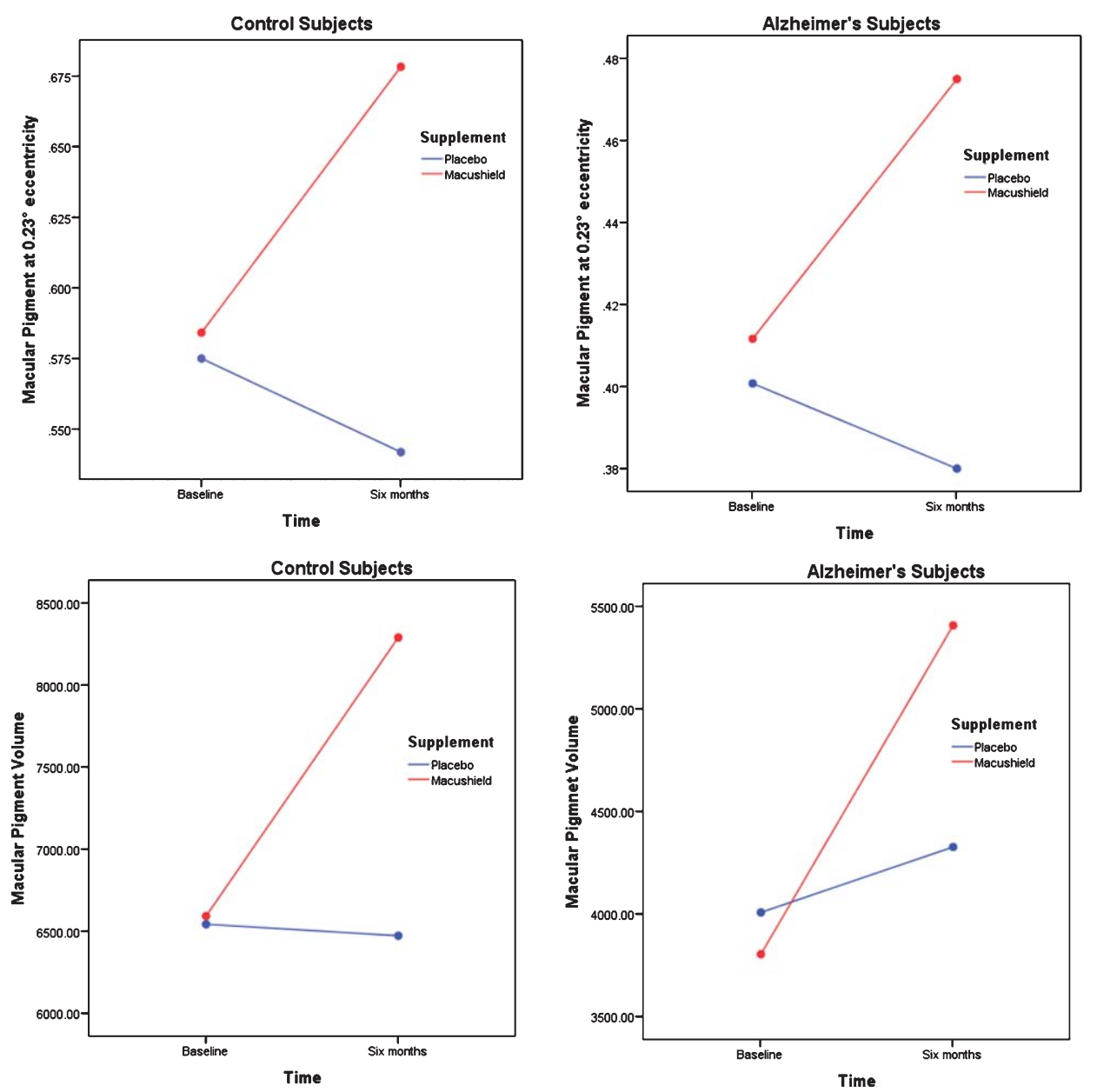

Fig. 2. Mean macular pigment at baseline and after six months of supplementation with either active supplement (Macushield) or placebo in subjects with Alzheimer's disease and control subjects. 
(Macushield) exhibit significantly increased MP, in both non-AD (control) and AD subjects, at six months. The placebo subjects exhibit no significant change over this time period.

\section{Visual function at baseline and after six months of supplementation}

\section{Best corrected visual acuity}

Repeated Measures Analysis of change in BCVA produced just one statistically significant effect, the Time*Supplement interaction effect $(p=0.005)$. Further examination shows that this effect arises because, unexpectedly, in both AD subjects and controls, average BCVA increased slightly with time in the placebo subjects, but declined in the Macushield subjects. Of note, however, the change observed here, although statistically significant, does not represent a meaningful change (clinically) in BCVA. Moreover, the paired samples $t$-test produced no significant results for either group, regardless of supplement ( $p>0.05$, for all).

\section{Contrast sensitivity}

In the Repeated Measures Analysis of change in $\mathrm{CS}$ at $1.2 \mathrm{cpd}$, the within-subjects Time*Supplement interaction effect was significant $(p<0.039)$. Neither the Time*Group interaction $(p=0.23)$ nor the Time*Supplement*Group interaction $(p=0.90)$ was significant. Thus, there was a significant increase in
$\mathrm{CS}$ at $1.2 \mathrm{cpd}$ after 6 months for subjects on the active supplement compared with subjects on the placebo supplement, no significant difference over time between AD and non-AD (control) subjects, and no evidence that the supplement worked differently over time for AD and non-AD (control) subjects.

No statistically significant findings were observed, from the Repeated Measures analysis, forCS at otherfrequencies. Examining the paired $t$-test results in Table 4 and Fig. 3, however, subjects on the active supplement exhibited four significant results (from five spatial frequencies tested) in the $\mathrm{AD}$ group, and two for the nonAD group, and all indicating improvements in CS.

\section{Cognitive function at baseline and after six months of supplementation}

We found no statistically significant main or interaction effects ( $p>0.05$, for all variables analyzed) from the Repeated Measures Analysis of any of the cognitive function outcome variables measured (see Table 1 for list of variables analyzed). Thus, supplementation with Macushield, over the six months of the study, did not significantly improve any of these cognitive function scores, in either AD or non-AD (control) subjects.

\section{DISCUSSION}

This report (CARDS 2) presents findings from a six-month macular carotenoid interventional, double-

Table 4

Contrast sensitivity at baseline and following six months of supplementation with either active or placebo intervention

\begin{tabular}{|c|c|c|c|c|c|c|}
\hline Group & Intervention & Measurement & mean $\pm \mathrm{SD}$ at baseline & mean \pm SD at 6 months & $\%$ Change & Sig. \\
\hline Control & placebo & $\mathrm{CS}$ at $1.2 \mathrm{cpd}$ & $1.83 \pm 0.154$ & $1.82 \pm 0.150$ & -0.01 & 0.84 \\
\hline Control & active & $\mathrm{CS}$ at $1.2 \mathrm{cpd}$ & $1.76 \pm 0.254$ & $1.88 \pm 0.249$ & 4.9 & 0.006 \\
\hline $\mathrm{AD}$ & placebo & $\mathrm{CS}$ at $1.2 \mathrm{cpd}$ & $1.51 \pm 0.273$ & $1.55 \pm 0.320$ & 2.9 & 0.108 \\
\hline $\mathrm{AD}$ & active & $\mathrm{CS}$ at $1.2 \mathrm{cpd}$ & $1.47 \pm 0.254$ & $1.63 \pm 0.237$ & 11 & 0.04 \\
\hline Control & placebo & $\mathrm{CS}$ at $2.4 \mathrm{cpd}$ & $1.81 \pm 0.180$ & $1.83 \pm 0.185$ & 1.1 & 0.6 \\
\hline Control & active & $\mathrm{CS}$ at $2.4 \mathrm{cpd}$ & $1.73 \pm 0.350$ & $1.82 \pm 0.290$ & 5 & 0.038 \\
\hline $\mathrm{AD}$ & placebo & $\mathrm{CS}$ at $2.4 \mathrm{cpd}$ & $1.47 \pm 0.420$ & $1.48 \pm 0.402$ & 0.7 & 0.461 \\
\hline $\mathrm{AD}$ & active & $\mathrm{CS}$ at $2.4 \mathrm{cpd}$ & $1.48 \pm 0.226$ & $1.55 \pm 0.241$ & 4.9 & 0.048 \\
\hline Control & placebo & $\mathrm{CS}$ at $6.0 \mathrm{cpd}$ & $1.37 \pm 0.240$ & $1.46 \pm 0.205$ & 3.3 & 0.275 \\
\hline Control & active & $\mathrm{CS}$ at $6.0 \mathrm{cpd}$ & $1.57 \pm 0.196$ & $1.59 \pm 0.161$ & 0.3 & 0.687 \\
\hline $\mathrm{AD}$ & placebo & $\mathrm{CS}$ at $6.0 \mathrm{cpd}$ & $1.34 \pm 0.263$ & $1.34 \pm 0.309$ & 0 & 0.785 \\
\hline $\mathrm{AD}$ & active & $\mathrm{CS}$ at $6.0 \mathrm{cpd}$ & $1.17 \pm 0.255$ & $1.29 \pm 0.303$ & 10 & 0.16 \\
\hline Control & placebo & $\mathrm{CS}$ at $9.6 \mathrm{cpd}$ & $1.16 \pm 0.286$ & $1.16 \pm 0.350$ & 0 & 0.919 \\
\hline Control & active & $\mathrm{CS}$ at $9.6 \mathrm{cpd}$ & $1.27 \pm 0.222$ & $1.32 \pm 0.171$ & 4 & 0.38 \\
\hline $\mathrm{AD}$ & placebo & $\mathrm{CS}$ at $9.6 \mathrm{cpd}$ & $1.03 \pm 0.266$ & $1.04 \pm 0.300$ & 0.9 & 0.84 \\
\hline $\mathrm{AD}$ & active & $\mathrm{CS}$ at $9.6 \mathrm{cpd}$ & $0.87 \pm 0.308$ & $1.00 \pm 0.340$ & 16 & 0.011 \\
\hline Control & placebo & $\mathrm{CS}$ at $15.15 \mathrm{cpd}$ & $0.89 \pm 0.31$ & $0.83 \pm 0.32$ & -7 & 0.39 \\
\hline Control & active & $\mathrm{CS}$ at $15.15 \mathrm{cpd}$ & $0.75 \pm 0.36$ & $0.88 \pm 0.25$ & 16 & 1.76 \\
\hline $\mathrm{AD}$ & placebo & $\mathrm{CS}$ at $15.15 \mathrm{cpd}$ & $0.85 \pm 0.16$ & $0.79 \pm 0.16$ & -7 & 0.471 \\
\hline $\mathrm{AD}$ & active & $\mathrm{CS}$ at $15.15 \mathrm{cpd}$ & $0.68 \pm 0.24$ & $0.85 \pm 0.24$ & 25 & 0.047 \\
\hline
\end{tabular}

Data displayed are mean \pm standard deviation. Sig., the $p$ value for paired-sample $t$ testing between baseline and six months for each group split by intervention; CS, contrast sensitivity; cpd, cycles per degree; AD, Alzheimer's disease; active, Macushield ${ }^{\mathrm{TM}}$ : 10 mg lutein, 10 mg meso-zeaxanthin, and $2 \mathrm{mg}$ zeaxanthin; placebo, sunflower oil. 

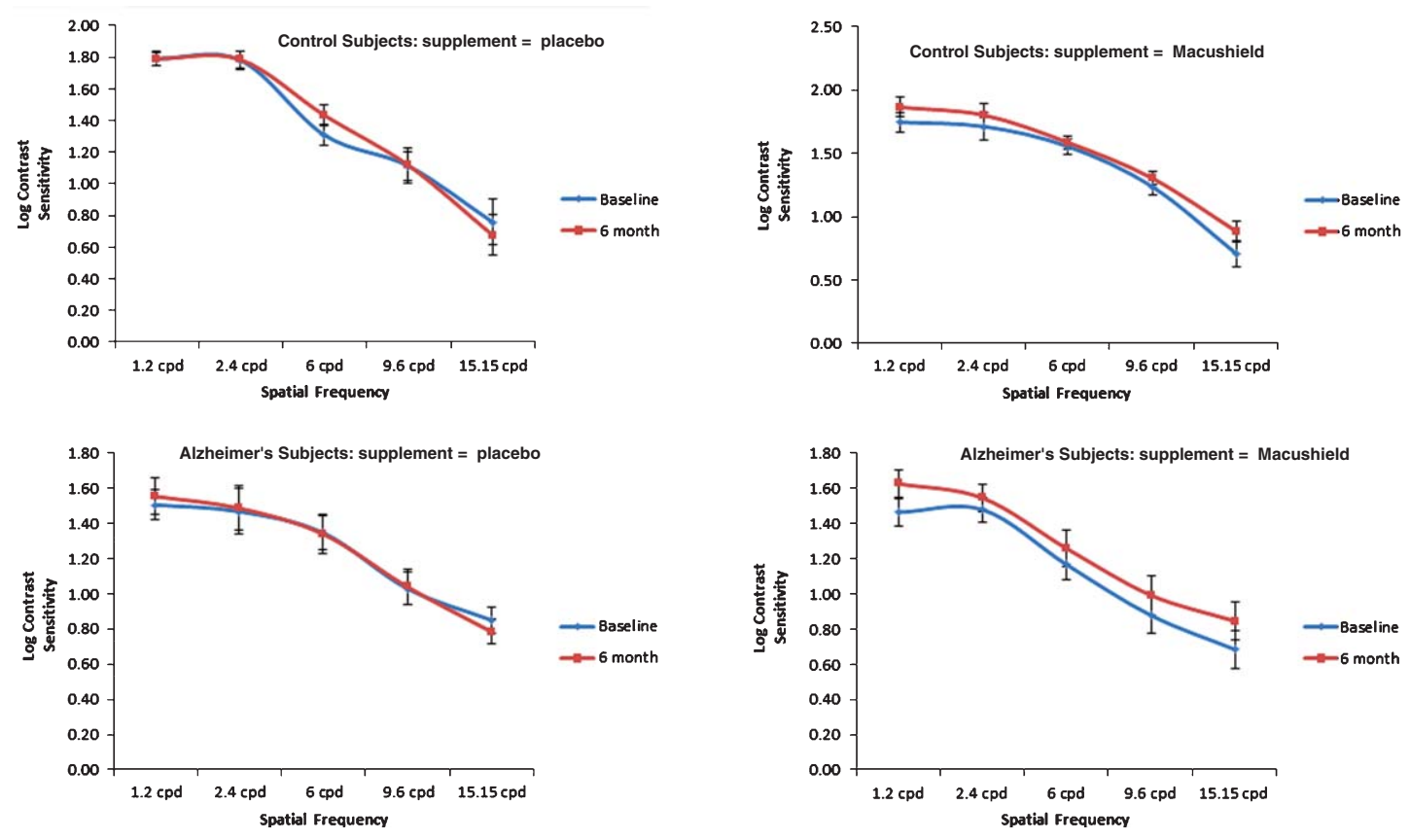

Fig. 3. Contrast sensitivity curve at baseline and after six months of supplementation with either active supplement (Macushield) or placebo in subjects with Alzheimer's disease and control subjects.

blind, placebo-controlled, randomized, clinical trial, in subjects with mild to moderate AD (AD subjects) compared with controls of similar age (non-AD control subjects). The rationale for conducting this experiment follows on from the previously reported finding that patients with moderate AD have significantly lower $\mathrm{MP}$, and significantly poorer visual function, when compared to control subjects of similar age. Also, given that enrichment of MP has been shown to improve visual function, in both diseased [12] and non-diseased retinae [11], it was logical to investigate whether a similar effect could be achieved in patients with $\mathrm{AD}$, where baseline visual function was sub-optimal. Of note, this is the first study of its kind to attempt to answer this important research question.

It is known that patients with dementia and AD have poor diets lacking in fruit and vegetables [47-49] and therefore we know that, on average, patients with $\mathrm{AD}$ consume less carotenoids than patients free of AD. Furthermore, it has been shown that high serum concentrations of $\mathrm{L}+\mathrm{Z}$ are associated with a lower risk of AD mortality in adults [50] and that plasma antioxidants are depleted in mild cognitive impairment and in $\mathrm{AD}$ when compared to subjects with normal cognitive function [51]. Indeed, our data is consistent with the above studies, as we confirm that (at baseline) patients with AD have significantly lower (33\% lower) dietary intake of foods known to contain the carotenoids ( $\mathrm{L}$ and Z) when compared to control subjects of comparable age. Also, we found that serum concentrations of $\mathrm{L}$ and $\mathrm{Z}$ were significantly lower in subjects with AD when compared to control subjects $(21 \%$ lower for $\mathrm{L}$ and $31 \%$ lower for $\mathrm{Z}$ ). These findings in diet and serum were reflected in the MP data, with AD subjects exhibiting significantly lower MP (28\% lower on average) when compared to control subjects. Finally, our data also confirms findings from our earlier publication [1], in that subjects with $\mathrm{AD}$ have significantly poorer vision when compared to the control subjects (e.g., for CS at $2.4 \mathrm{cpd}$, subjects with $\mathrm{AD}$ have lower CS [17.9\%] when compared to controls).

The main findings from our study are that $\mathrm{AD}$ sufferers who were supplemented with a carotenoid formulation containing $10 \mathrm{mg} \mathrm{MZ}, 10 \mathrm{mg} \mathrm{L}$, and $2 \mathrm{mg}$ of $\mathrm{Z}$, exhibited significant increases in serum concentrations of MZ, L, and Z, and in MP, with consequential improvements in visual function (in terms of CS); whereas, the placebo groups exhibited no significant change in any of these outcome measures. Of note, the increases observed in MP (and serum concentrations of its constituent carotenoids) were comparable between $\mathrm{AD}$ and non-AD (control) subjects. Indeed, at six months, subjects receiving the active intervention (10 mg MZ, $10 \mathrm{mg} \mathrm{L}$, and $2 \mathrm{mg} \mathrm{Z}$ ) were comparable 
in terms of average circulating serum concentrations of $\mathrm{L}, \mathrm{Z}$, and $\mathrm{MZ}$, irrespective of whether they were in the AD or non-AD (control) groups, with no significant difference between these groups for any of these carotenoids at this point ( $p>0.05$ for all comparisons). The importance of this finding rests on the logical conclusion that the observed and relative lack of circulating serum carotenoid concentrations and MP in $\mathrm{AD}$ [1] is not attributable to an inability of these patients to respond to carotenoid intake (e.g. they are probably not compromised in terms of carotenoid absorption, transport, or uptake). In other words, the findings are consistent with the view that the reason why patients with AD have lower MP compared to control subjects is likely due to an associated poor dietary intake of foods containing carotenoids (fruits and vegetables).

With respect to the serum and MP response exhibited in both $\mathrm{AD}$ and non-AD (control) groups, our data is consistent with previous studies where a supplement containing all three of the macular carotenoids (10 mgMZ, $10 \mathrm{mgL}$, and $2 \mathrm{mgZ}$ ) was used [11, 12, $52,53]$. Indeed, it is noteworthy from previous studies that carotenoid supplements that do not contain MZ in their formulation did not augment MP significantly at six months [38, 54]. It appears, therefore, that best results in terms of increasing serum carotenoid concentrations (for MZ, L, and Z), and MP augmentation, is achieved when all three of the macular carotenoids are included in the formulation, and this observation also holds true for patients with AD. Further, supplementation with macular carotenoids, and consequential MP augmentation, is associated with risk reduction for AMD, a particularly important benefit as intervention with current treatment modalities (i.e., monthly injections, under local anesthesia, into the eye) would be problematic in this patient group.

We believe that it is important to draw attention to our findings pertaining to visual function. Firstly, we confirm that CS is significantly lower in AD subjects when compared to non-AD controls. Addressing this sensory defect in vulnerable $\mathrm{AD}$ patients should be a priority for those involved in the care of patients with this form of cognitive impairment, and routine and frequent assessment of visual function should be incorporated into the delivery of that care. For example, improvements seen among AD subjects supplemented with $\mathrm{MZ}, \mathrm{L}$, and $\mathrm{Z}$ were clinically meaningful at spatial frequencies of $1.2 \mathrm{cpd}$ and $15.15 \mathrm{cpd}$, equating to approximately one line of improvement on standard Pelli-Robson chart, and likely to enhance visual appreciation of small and large targets by these subjects. We suggest that further studies may con- sider other measures of visual function (e.g., glare disability and photostress recovery), however, the feasibility of including these measures will need to be considered given the time required to perform the tests and the ability of the subject to perform each test.

Of note, no improvements in cognitive function were demonstrated as a result of supplementation in either $\mathrm{AD}$ or non-AD control subjects, a finding that is neither surprising nor counter-intuitive. The rationale whereby antioxidants are important for cognition rests on their ability to prevent or attenuate oxidative damage, as opposed to tissue repair. In other words, there is a biologically plausible rationale, supported by emerging evidence, that antioxidant intake is protective for cognition, but the notion that established cognitive impairment could be reversed by supplementation with antioxidants is less probable, especially in the context of a short period of intervention (as reported herein). Therefore, to investigate properly if supplementation with the carotenoids L, Z, and MZ impact positively on cognitive health/function, we suggest that subjects with very early signs of cognitive decline, and subjects of comparable age with no signs of cognitive decline are selected, and are followed for at least 3 years. The current study confirms that AD patients respond to carotenoid supplements in the same way as normal controls, and therefore it is possible that supplementation with these nutrients, if achieved early enough, may support and protect cognitive health.

In conclusion, our data suggests that supplementation with the macular carotenoids (MZ, Z, and L) may benefit patients with $\mathrm{AD}$, in terms of clinically meaningful improvements in visual function and in terms of MP augmentation (and consequential risk reduction for AMD). The impact of sustained supplementation on cognition and visual function in $\mathrm{AD}$ subjects, and on risk for $\mathrm{AD}$, both warrant further study.

\section{ACKNOWLEDGMENTS}

We would like to thank the Howard Foundation, Cambridge, CB22 5LA, United Kingdom for supporting this research. We would like to acknowledge Cambridge Cognition, UK for guidance with respect to the assessment of cognitive function. Also, we would like to thank all the staff at the UHW, Age-Related Care Unit and at the Vision Research Centre, Waterford Institute of Technology for assisting this study.

The Principal Investigator (Professor John M Nolan) is supported by the European Research Council (ERC) (CREST 281096). 
Authors' disclosures available online (http://www.jalz.com/disclosures/view.php?id=2602).

\section{REFERENCES}

[1] Nolan JM, Loskutova E, Howard AN, Moran R, Mulcahy R, Stack J, Bolger M, Dennison J, Akuffo KO, Owens N, Thurnham DI, Beatty S (2014) Macular pigment, visual function, and macular disease among subjects with Alzheimer's disease: An exploratory study. J Alzheimers Dis $\mathbf{4 2}$, 1191-1202.

[2] Bone RA, Landrum JT, Fernandez L, Tarsis SL (1988) Analysis of the macular pigment by HPLC: Retinal distribution and age study. Invest Ophthalmol Vis Sci 29, 843-849.

[3] Wooten BR, Hammond BR, Land RI, Snodderly DM (1999) A practical method for measuring macular pigment optical density. Invest Ophthalmol Vis Sci 40, 2481-2489.

[4] Dennison JL, Stack J, Beatty S, Nolan JM (2013) Concordance of macular pigment measurements obtained using customized heterochromatic flicker photometry, dual-wavelength autofluorescence, and single-wavelength reflectance. Exp Eye Res 116, 190-198.

[5] Hirsch J, Curcio CA (1989) The spatial resolution capacity of human foveal retina. Vision Res 29, 1095-1101.

[6] Snodderly DM, Brown PK, Delori FC, Auran JD (1984) The macular pigment. I. absorbance spectra, localization, and discrimination from other yellow pigments in primate retinas. Invest Ophthalmol Vis Sci 25, 660-673.

[7] Khachik F, Bernstein PS, Garland DL (1997) Identification of lutein and zeaxanthin oxidation products in human and monkey retinas. Invest Ophthalmol Vis Sci 38, 1802-1811.

[8] Li B, Ahmed F, Bernstein PS (2010) Studies on the singlet oxygen scavenging mechanism of human macular pigment. Arch Biochem Biophys 504, 56-60.

[9] Chew EY, Clemons TE, SanGiovanni JP, Danis RP, Ferris FL III, Elman MJ, Antoszyk AN, Ruby AJ, Orth D, Bressler SB, Fish GE, Hubbard GB, Klein ML, Chandra SR, Blodi BA, Domalpally A, Friberg T, Wong WT, Rosenfeld PJ, Agron E, Toth CA, Bernstein PS, Sperduto RD (2013) Secondary analyses of the effects of lutein/zeaxanthin on age-related macular degeneration progression: AREDS2 report No. 3. JAMA Ophthalmol 132, 142-149.

[10] Loughman J, Akkali MC, Beatty S, Scanlon G, Davison PA, O'Dwyer V, Cantwell T, Major P, Stack J, Nolan JM (2010) The relationship between macular pigment and visual performance. Vision Res 50, 1249-1256.

[11] Loughman J, Nolan JM, Howard AN, Connolly E, Meagher K, Beatty S (2012) The impact of macular pigment augmentation on visual performance using different carotenoid formulations. Invest Ophthalmol Vis Sci 53, 7871-7880.

[12] Sabour-Pickett S, Beatty S, Connolly E, Loughman J, Stack J, Howard A, Klein R, Klein BE, Meuer SM, Myers CE, Akuffo KO, Nolan JM (2014) Supplementation with three different macular carotenoid formulations in patients with early agerelated macular degeneration. Retina 34, 1757-1766.

[13] Huang YM, Yan SF, Ma L, Zou ZY, Xu XR, Dou HL, Lin XM (2013) Serum and macular responses to multiple xanthophyll supplements in patients with early age-related macular degeneration. Nutrition 29, 387-392.

[14] Murray IJ, Makridaki M, van der Veen RL, Carden D, Parry NR, Berendschot TT (2013) Lutein supplementation over a one-year period in early AMD might have a mild beneficial effect on visual acuity: The CLEAR study. Invest Ophthalmol Vis Sci 54, 1781-1788.

[15] Weigert G, Kaya S, Pemp B, Sacu S, Lasta M, Werkmeister RM, Dragostinoff N, Simader C, Garhofer G, Schmidt-Erfurth U, Schmetterer L (2011) Effects of lutein supplementation on macular pigment optical density and visual acuity in patients with age-related macular degeneration. Invest Ophthalmol Vis Sci 52, 8174-8178.

[16] Wooten BR, Hammond BR (2002) Macular pigment: Influences on visual acuity and visibility. Prog Retin Eye Res $\mathbf{2 1}$, 225-240.

[17] Craft NE, Haitema TB, Garnett KM, Fitch KA, Dorey CK (2004) Carotenoid, tocopherol, and retinol concentrations in elderly human brain. J Nutr Health Aging 8, 156-162.

[18] Vishwanathan R, Neuringer M, Snodderly DM, Schalch W, Johnson EJ (2013) Macular lutein and zeaxanthin are related to brain lutein and zeaxanthin in primates. Nutr Neurosci 16, 21-29.

[19] Johnson EJ, Vishwanathan R, Johnson MA, Hausman DB, Davey A, Scott TM, Green RC, Miller LS, Gearing M, Woodard J, Nelson PT, Chung HY, Schalch W, Wittwer J, Poon LW (2013) Relationship between serum and brain carotenoids, alpha-tocopherol, and retinol concentrations and cognitive performance in the oldest old from the Georgia Centenarian Study. J Aging Res 2013, 951786.

[20] Vishwanathan R, Iannaccone A, Scott TM, Kritchevsky SB, Jennings BJ, Carboni G, Forma G, Satterfield S, Harris T, Johnson KC, Schalch W, Renzi LM, Rosano C, Johnson EJ (2014) Macular pigment optical density is related to cognitive function in older people. Age Ageing 43, 271-275.

[21] Renzi LM, Dengler MJ, Puente A, Miller LS, Hammond BR Jr (2014) Relationships between macular pigment optical density and cognitive function in unimpaired and mildly cognitively impaired older adults. Neurobiol Aging 35, 1695-1699.

[22] Feeney J, Finucane C, Savva GM, Cronin H, Beatty S, Nolan JM, Kenny RA (2013) Low macular pigment optical density is associated with lower cognitive performance in a large, population-based sample of older adults. Neurobiol Aging 34, 2449-2456.

[23] Johnson EJ, McDonald K, Caldarella SM, Chung HY, Troen AM, Snodderly DM (2008) Cognitive findings of an exploratory trial of docosahexaenoic acid and lutein supplementation in older women. Nutr Neurosci 11, 75-83.

[24] Keller JN, Schmitt FA, Scheff SW, Ding Q, Chen Q, Butterfield DA, Markesbery WR (2005) Evidence of increased oxidative damage in subjects with mild cognitive impairment. Neurology 64, 1152-1156.

[25] Teunissen CE, van Boxtel MP, Bosma H, Bosmans E, Delanghe J, De BC, Wauters A, Maes M, Jolles J, Steinbusch HW, de Vente J (2003) Inflammation markers in relation to cognition in a healthy aging population. J Neuroimmunol 134, 142-150.

[26] Pappolla MA, Smith MA, Bryant-Thomas T, Bazan N, Petanceska S, Perry G, Thal LJ, Sano M, Refolo LM (2002) Cholesterol, oxidative stress, and Alzheimer's disease: Expanding the horizons of pathogenesis. Free Radic Biol Med 33, 173-181.

[27] Wyss-Coray T (2006) Inflammation in Alzheimer disease: Driving force, bystander or beneficial response? Nat Med 12, 1005-1015.

[28] Ciccone MM, Cortese F, Gesualdo M, Carbonara S, Zito A, Ricci G, De Pascalis F, Scicchitano P, Riccioni G (2013) Dietary intake of carotenoids and their antioxidant and antiinflammatory effects in cardiovascular care. Mediators Inflam 2013, 782137. 
[29] Kijlstra A, Tian Y, Kelly ER, Berendschot TT (2012) Lutein: More than just a filter for blue light. Prog Retin Eye Res 31, 303-315.

[30] Johnson EJ (2012) A possible role for lutein and zeaxanthin in cognitive function in the elderly. Am J Clin Nutr 96, 1161S$1165 \mathrm{~S}$.

[31] Stahl W, Sies H (2001) Effects of carotenoids and retinoids on gap junctional communication. Biofactors 15, 95-98.

[32] Stahl W, Nicolai S, Briviba K, Hanusch M, Broszeit G, Peters M, Martin HD, Sies H (1997) Biological activities of natural and synthetic carotenoids: Induction of gap junctional communication and singlet oxygen quenching. Carcinogenesis 18, 89-92.

[33] Strauss E, Scherman EMS, Spreen O (2006) A Compendium of Neuropsychological Tests. Administration, Norms and Commentary, Oxford University Press; New York.

[34] Robbins TW, James M, Owen AM, Sahakian BJ, McInnes L, Rabbitt P (1994) Cambridge Neuropsychological Test Automated Battery (CANTAB): A factor analytic study of a large sample of normal elderly volunteers. Dementia 5, 266-281.

[35] Sahakian BJ, Morris RG, Evenden JL, Heald A, Levy R, Philpot M, Robbins TW (1988) A comparative study of visuospatial memory and learning in Alzheimer-type dementia and Parkinson's disease. Brain 111(Pt 3), 695-718.

[36] Owen AM, Downes JJ, Sahakian BJ, Polkey CE, Robbins TW (1990) Planning and spatial working memory following frontal lobe lesions in man. Neuropsychologia 28, 1021-1034

[37] Charalampidou S, Nolan J, Loughman J, Stack J, Higgins G, Cassidy L, Beatty S (2011) Psychophysical impact and optical and morphological characteristics of symptomatic non-advanced cataract. Eye (Lond) 25, 1147-1154.

[38] Nolan JM, Loughman J, Akkali MC, Stack J, Scanlon G, Davison P, Beatty S (2011) The impact of macular pigment augmentation on visual performance in normal subjects: COMPASS. Vision Res 51, 459-469.

[39] Akuffo KO, Beatty S, Stack J, Dennison J, O'Regan S, Meagher KA, Peto T, Nolan J (2014) Central Retinal Enrichment Supplementation Trials (CREST): Design and methodology of the CREST randomized controlled trials. Ophthalmic Epidemiol 21, 111-123.

[40] Bird AC, Bressler NM, Bressler SB, Chisholm IH, Coscas G, Davis DM, de Jong PT, Klaver CC, Klein BE, Klein R, et al. (1995) An international classification and grading system for age-related maculopathy and age-related macular degeneration. The International ARM Epidemiological Study Group. Surv Ophthalmol 39, 367-374.

[41] Neelam K, Muldrew A, Hogg R, Stack J, Chakravarthy U, Beatty S (2009) Grading of age-related maculopathy: Slit- lamp biomicroscopy versus an accredited grading center. Retina 29, 192-198.

[42] Delori FC (2004) Autofluorescence method to measure macular pigment optical densities fluorometry and autofluorescence imaging. Arch Biochem Biophys 430, 156-162.

[43] Perry A, Rasmussen H, Johnson EJ (2009) Xanthophyll (lutein, zeaxanthin) content in fruits, vegetables and corn and egg products. J Food Compost Anal 22, 9-15.

[44] Thurnham DI, Tremel A, Howard AN (2008) A supplementation study in human subjects with a combination of meso-zeaxanthin, (3R,3'R)-zeaxanthin and (3R,3'R,6'R)lutein. Br J Nutr 100, 1307-1314.

[45] Hintze J (2008) PASS 2008. NCSS LLC, http://www. ncss.com

[46] Cohen J (1988) Statistical Power Analysis for the Behavioral Sciences, Lawrence Erlbaum Associates, Hillsdale, New Jersey.

[47] Morley JE (2010) Nutrition and the brain. Clin Geriatr Med 26, 89-98.

[48] Salerno-Kennedy R, Cashman KD (2007) The relationship between nutrient intake and cognitive performance in people at risk of dementia. Ir J Med Sci 176, 193-198.

[49] Mi W, van Wijk N, Cansev M, Sijben JW, Kamphuis PJ (2013) Nutritional approaches in the risk reduction and management of Alzheimer's disease. Nutrition 29, 1080-1089.

[50] Min JY, Min KB (2014) Serum lycopene, lutein and zeaxanthin, and the risk of Alzheimer's disease mortality in older adults. Dement Geriatr Cogn Disord 37, 246-256.

[51] Rinaldi P, Polidori MC, Metastasio A, Mariani E, Mattioli P, Cherubini A, Catani M, Cecchetti R, Senin U, Mecocci P (2003) Plasma antioxidants are similarly depleted in mild cognitive impairment and in Alzheimer's disease. Neurobiol Aging 24, 915-919.

[52] Meagher KA, Thurnham DI, Beatty S, Howard AN, Connolly E, Cummins W, Nolan JM (2013) Serum response to supplemental macular carotenoids in subjects with and without age-related macular degeneration. Br J Nutr 110, 289-300.

[53] Nolan JM, Akkali MC, Loughman J, Howard AN, Beatty S (2012) Macular carotenoid supplementation in subjects with atypical spatial profiles of macular pigment. Exp Eye Res 101, 9-15.

[54] Beatty S, Chakravarthy U, Nolan JM, Muldrew KA, Woodside JV, Denny F, Stevenson MR (2013) Secondary outcomes in a clinical trial of carotenoids with coantioxidants versus placebo in early age-related macular degeneration. Ophthalmology 120, 600-606. 